Pacific

Journal of

Mathematics

\title{
THE SEMIGROUP OF A SPACE CURVE SINGULARITY
}

Julio CASTELlanos 


\title{
THE SEMIGROUP OF A SPACE CURVE SINGULARITY
}

\author{
Julio Castellanos
}

\begin{abstract}
The semigroup of values of a space curve singularity is an invariant of the singularity. We analyze the complexity of this invariant, in order to describe the geometric invariants of the sequence of infinitely near points of the curve necessary to determine the generators of the semigroup. We give several approaches and examples using Hamburger-Noether matrices to describe the infinitely near points of the curve.
\end{abstract}

\section{Introduction}

In the Zariski equisingularity theory [Zariski 1965] of plane curve singularities, one of the invariants considered for irreducible curves is the semigroup of values. An irreducible algebroid curve (or branch) $C$ over an algebraically closed field $k$, with ring $R$, has a normalization isomorphic to $k \llbracket t \rrbracket$, and the injection $R \hookrightarrow k \llbracket t \rrbracket$ provides a valuation and a semigroup of values for $C$. The graded ring associated with the valuation is the ring of a monomial curve having the same semigroup as the given branch.

For plane branch singularities this graded ring was studied by Lejeune-Jalabert and Teissier [Lejeune-Jalabert 1973; Lejeune-Jalabert and Teissier 1975; Teissier 1973]. The minimal system of generators for the semigroup is formed by the intersection multiplicities of the branch with the high maximal contact curves or, from a different point of view, Abhyankar's approximate roots [Abhyankar and Moh 1973a; 1973b; Popescu-Pampu 2003]. Goldin and Teissier [2000] use the embedding of a plane branch into a $(g+1)$-dimensional space (where $g$ is the number of generators of the semigroup) in order to obtain a resolution of the singularity of branch in one toric modification. They consider this approach as the first step of a general method for any singularity, in order to get a Zariski local uniformization theorem for arbitrary valuations.

For plane branch singularities the semigroup of values is determined by means of the characteristic exponents, that is, by the proximity relations of the infinitely near points of the resolution of the branch. For space curve singularities this is not

MSC2000: 14B05, 14H2D.

Keywords: curve singularities, numerical semigroups.

Supported by DGICYT grant BFM2001-2251. 
the case, and the semigroup of values is much more complicated than for plane branch singularities. It is not currently known how to obtain the semigroup of values from the geometry of the resolution of the curve.

Our aim in this article is to determine some facts about the geometry of the resolution of a space branch singularity, which will then allow us to compute the semigroup of values. We also aim to find the hypersurfaces that provide the generators of the semigroup of values, which generalize "high maximal contact curves" and "approximate roots".

In Section 1 we describe the infinitely near points associated to any resolution of the singularities of a curve, and we present the Hamburger-Noether matrices associated with them. These matrices are like analytic coordinates of the infinitely near points, and give us a parametrization for the branches different to the Puiseux one. In Section 2 we describe different semigroups: the semigroup of values, the Arf semigroup, and the semigroup associated with the characteristic exponents.

In Section 3 we consider the linear proximity of infinitely near points of dimension $l$, a generalization of the proximity for the plane case, which provides elements of the semigroup of values. In Section 4 we consider higher degree proximity and hypersurfaces of maximal contact and we introduce the shadow clusters, a kind of closure cluster of the ones given by some weights over infinitely near points. We consider the idealistic semigroup, associated with the special complete ideals for sequences of infinitely near points (considered by Lipman [1988]) and relate it to the semigroup of values.

Throughout the work we present several examples. To compute the examples we have used a Maple implementation of an algorithm for computing the semigroup of values of a space curve singularity established in [Castellanos and Castellanos 2005].

\section{Space curve singularities}

We consider a space curve singularity to be an algebroid curve (or simply curve) $C=\operatorname{Spec}(R)$, where $(R, \mathfrak{m}, k)$ is a complete equicharacteristic noetherian local ring of dimension 1 containing an algebraic closed field $k$ as a coefficient field. By Cohen's theorem, if $\left\{x_{1}, \ldots x_{N}\right\}$ is a system of generators of $\mathfrak{m}$, it follows that

$$
R \simeq k \llbracket X_{1}, \ldots, X_{N} \rrbracket / \mathfrak{p},
$$

and we say that the curve $C$ is embedded in the $N$-space $V=\operatorname{Spec}(A)$, where $A=k \llbracket X_{1}, \ldots, X_{N} \rrbracket$. The embedding dimension (embdim) is the minimum possible $N$, which is equal to $\operatorname{dim}_{k} \mathfrak{m} / \mathfrak{m}^{2}$. For $N=2$ we say that $C$ is a plane curve singularity. In the following we consider only irreducible curves, that is, where $R$ is a domain. 
The integer closure $\bar{R}$ of $R$ in its quotient field is a discrete ring valuation, and $R \hookrightarrow \bar{R} \simeq k \llbracket t \rrbracket$, that is, each $z \in R$ gives a series $z(t)$. So $R \simeq k \llbracket x_{1}(t), \ldots, x_{N}(t) \rrbracket$ and we call $\left\{x_{1}(t), \ldots, x_{N}(t)\right\}$ a parametrization for $C$. The multiplicity of the curve $C$ is the multiplicity $n=e(R)$ of the local ring, and is the minimum order of the series of any parametrization of the curve. The multiplicity sequence $E(C)$ of $C$ is $\left\{n=n_{0}, \ldots, n_{i}, \ldots, n_{r}=1\right\}$, where $n_{i}=e\left(R^{(i)}\right)$ is the multiplicity of the $i$-th blowing up of $R$ in its maximal ideal, $R^{(i)}=\mathrm{B}_{\mathfrak{m}_{i-1}}\left(R^{(i-1)}\right)$.

We consider below another kind of parametrization for the curve, the so-called Hamburger-Noether matrices (see [Castellanos 1995]) associated with the infinitely near points described by the embedded resolution of the curve singularity.

Let $P \in V$ be the closed point. Denote by $V_{1}$ the blowing up of $V$ with center $P$, and by $E_{1} \simeq \mathbb{P}^{N-1}$ the exceptional divisor. An infinitely near point (INP) to $P$ in the first neighborhood is defined to be a point $Q$ in the exceptional divisor of $V_{1}$. A sequence or chain of INPs of $P$ is a set $\left\{P, P_{1}, \ldots, P_{i}, \ldots, P_{s}\right\}$, where $P_{0}=P$ and each $P_{i}$ (for $i=1, \ldots, s$ ) is a closed point in $E_{i} \subset V_{i}$, the exceptional divisor of the blowing up $\pi_{i}: V_{i} \rightarrow V_{i-1}$ at $P_{i-1}$, where $V_{0}=V$, and $P_{i}$ is an INP to $P$ in the $i$-th neighborhood.

We consider an embedded resolution of the curve singularity of $C$ in $V$, not necessarily minimal. That is, we make a sequence of blowing ups and we do not necessarily stop when we get curves with multiplicity one. That process provides a sequence of INPs of the closed point $P$ of $V$. That sequence can be infinite. Let $\pi_{1}: V_{1} \rightarrow V$ be the blowing-up of $V$ at $P$. Let $C_{1}$ be the strict transform of $C$ by $\pi_{1}$. The tangent cone of $C$ is given by a line $L_{1}$ that determines a point $P_{1}$ in the curve $C_{1}$ lying in the exceptional divisor $E_{1}$ of $\pi_{1}$. The embedded $C \hookrightarrow V$ gives one between the blowing-ups of $C$ and $V$ at $P$,

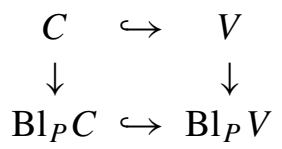

Then for all $i \geq 1$ we have the diagram

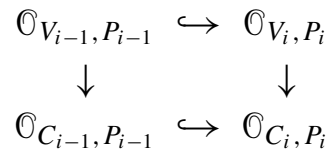

which yields the diagram

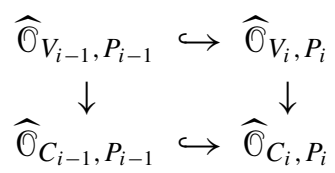


for the (maximal ideal)-adic completions of their topologies. Set $R_{i-1}=\widehat{\widehat{O}}_{C_{i-1}, P_{i-1}}$; then since

$$
\widehat{\mathrm{O}}_{V_{i-1}, P_{i-1}} \simeq k \llbracket X_{1}^{(i-1)}, \ldots, X_{N}^{(i-1)} \rrbracket,
$$

we have $R_{i-1} \simeq k \llbracket X_{1}^{(i-1)}, \ldots, X_{N}^{(i-1)} \rrbracket / \mathfrak{p}_{i}$, and we obtain a sequence $\left\{P_{i}: i \geq 0\right\}$ of INPs. The coordinates of each point $P_{i} \in \mathbb{E}_{i}$ correspond to the direction of the tangent line to $C_{i-1}$ at $P_{i-1}$, and they are denoted by $\left(a_{i 1}: \cdots: 1^{(j)}: \cdots: a_{i N}\right)$, where $1^{(j)}$ denotes a 1 in the $j$-th index, and $R_{i}$ is obtained by

$$
R_{i-1}\left[x_{1}^{(i-1)} / x_{j}^{(i-1)}, \ldots, 1^{(j}, \ldots, x_{N}^{(i-1)} / x_{j}^{(i-1)}\right] \simeq \widehat{\widehat{O}}_{C_{i}, P_{i}} .
$$

By the properties of the conductor ideal of $C$ only a finite number of INPs associated with $C$ determine the curve, as we will see in Remark 2.2.

Conversely, any sequence of INPs of $P$ provides a unique irreducible curve singularity, the so-called generic curve, by passing through the sequence in a linear sense; see [Castellanos and Nuñez 1991].

Given a parametrization $\left\{x_{(i-1) 1}(t), \ldots, x_{(i-1) N}(t)\right\}$ of $C_{i-1}$, the expression

$$
\left\{x_{(i-1) 1}(t),\left(x_{(i-1) 2}(t) / x_{(i-1) 1}(t)\right)-b_{i 2}, \ldots,\left(x_{(i-1) N}(t) / x_{(i-1) 1}(t)\right)-b_{i N}\right\}
$$

is a parametrization for the quadratic transform $C_{i}$ (blowing up + localization + completion), provided that $o_{t}\left(x_{(i-1) 1}(t)\right)$ is minimal for all $j \in\{1, \ldots, N\}$ and that the $b_{i j} \in k$ are chosen so that $o_{t}\left(\left(x_{(i-1) j}(t) / x_{(i-1) 1}(t)\right)-b_{i j}\right)>0$.

For each sequence $\left\{P, P_{1}, \ldots, P_{s}, \ldots\right\}$ of INPs of the curve, we may construct a matrix, the Hamburger-Noether $(\mathrm{H}-\mathrm{N})$ matrix $H$, with entries in the field $k$, consisting of $N$ rows and an infinite number of columns, each one given by an INP

Consider a parameter system $\left\{x_{1}, \ldots, x_{N}\right\}$ for the maximal ideal $\mathfrak{m}$, and let $\left(a_{1}: \cdots: a_{N}\right)$ be the coordinates of the direction corresponding to $L_{1}$, that is, $P_{1}$ in the exceptional divisor $E_{1}$. Suppose that the coordinate $a_{1}$ is nonzero, then the quadratic transformation of $V$ at $P_{1}$ is given by

$$
\begin{aligned}
T_{1}: R & \rightarrow \widehat{\mathcal{O}}_{\mathrm{B} l_{\mathfrak{M}}(R), P_{1}}=R_{1} \\
x_{1} & \mapsto x_{1}^{(1)} \\
x_{i} & \mapsto x_{i}^{(1)} x_{1}^{(1)}+a_{i 1} x_{1}^{(1)}, \quad \text { for } i=2, \ldots, N
\end{aligned}
$$

since $P_{1}$ is placed in the chart where the transform of $x_{1}$ divides the transforms of the other $x_{i}$.

Inductively, for each point $P_{i}$ of the sequence of INPs, we have coordinates $\left(a_{1 i}: \cdots: a_{(j-1) i}: 1: a_{(j+1) i}: \cdots: a_{N i}\right)$, if $P_{i}$ is placed in the chart given by $x_{j}^{(i)} \neq 0$.

The Hamburger matrix is composed of boxes as follows: 
(i) Suppose that $P_{1}, \ldots, P_{h}$, can be chosen in the chart where the transform of $x_{1}$ divides the transforms of the other $x_{i}$ and $P_{h+1}$ cannot. Then the first box is:

$$
B_{0}\left|\begin{array}{cccc}
\mathbf{1} & \mathbf{0} & \ldots & \mathbf{0} \\
a_{21} & a_{22} & \ldots & a_{2 h} \\
\vdots & \vdots & \vdots & \vdots \\
a_{N 1} & a_{N 2} & \ldots & a_{N h}
\end{array}\right|
$$

(ii) Now there is an index $q$, not necessarily unique, with $2 \leq q \leq N$ such that $P_{h+1}$ can be chosen in the chart given by $x_{q}^{(h+1)} \neq 0$. Choose one of these indices, say $q$, and suppose that $P_{h+1}, \ldots, P_{h+h_{1}}$ are such that they can be chosen in the chart given by $x_{q}^{(h+i)}$ and $P_{h+h_{1}+1}$ cannot. Then the second box of the matrix is:

$$
B_{1}\left|\begin{array}{cccc}
0 & a_{12}^{1} & \ldots & a_{1 h_{1}}^{1} \\
\vdots & \vdots & \vdots & \vdots \\
\mathbf{1} & \mathbf{0} & \ldots & \mathbf{0} \\
\vdots & \vdots & \vdots & \vdots \\
a_{N 1}^{1} & a_{N 2}^{1} & \ldots & a_{N h_{1}}^{1}
\end{array}\right| \leftarrow q
$$

(iii) Now repeat this process as many times as needed and the final result of the matrix is:

$$
\left(\begin{array}{cccc|cccc}
\mathbf{1} & \mathbf{0} & \ldots & \mathbf{0} & 0 & a_{12}^{1} & \ldots & a_{1 h_{1}}^{1} \\
a_{21} & a_{22} & \ldots & a_{2 h} & a_{21}^{1} & a_{22}^{1} & \ldots & a_{2 h_{1}}^{1} \\
\vdots & \vdots & \vdots & \vdots & \vdots & \vdots & \vdots & \vdots \\
\vdots & \vdots & \vdots & \vdots & \vdots & \vdots & \vdots & \vdots \\
a_{q 1} & a_{q 2} & \ldots & a_{q h} & \mathbf{1} & \mathbf{0} & \ldots & \mathbf{0} \\
\vdots & \vdots & \vdots & \vdots & \vdots & \vdots & \vdots & \vdots \\
a_{N 1} & a_{N 2} & \ldots & a_{N h} & a_{N 1}^{1} & a_{N 2}^{1} & \ldots & a_{N h_{1}}^{1}
\end{array}|\ldots| \begin{array}{cccc|ccc}
a_{12}^{t-1} & a_{12}^{t-1} & \ldots & a_{1 h_{t-1}}^{t-1} & a_{11}^{t} & a_{12}^{t} & \ldots \\
\vdots & \vdots & \vdots & \vdots & \vdots & \vdots & \vdots \\
a_{i 1}^{t-1} & a_{i 2}^{t-1} & \ldots & a_{i h_{t-1}}^{t-1} & \mathbf{1} & \mathbf{0} & \ldots \\
\vdots & \vdots & \vdots & \vdots & \vdots & \vdots & \vdots \\
\mathbf{1} & \mathbf{0} & \ldots & \mathbf{0} & 0 & a_{s 2}^{t} & \ldots \\
\vdots & \vdots & \vdots & \vdots & \vdots & \vdots & \vdots \\
a_{N 1}^{t-1} & a_{N 2}^{t-1} & \ldots & a_{N h_{t-1}}^{t-1} & a_{N 1}^{t} & a_{N 2}^{t} & \ldots
\end{array}\right)
$$

The matrix has a marked row $\mathbf{1}, \mathbf{0}, \ldots, \mathbf{0}$ in each box, and the entry following this row is zero.

The marked row in each box is not an invariant of the sequence of INPs since it depends on the choice of the chart in the above procedure. From now on, we make this choice according to the following rule.

Rule. If there are several possibilities to mark the rows, and some of them have already been marked, then we choose one of them, precisely the last one marked. If all of them are new, then we choose any of them. 
So, for each H-N matrix $H$ of a sequence of INPs we get a sequence of marked rows, $I(H)=\left\{i_{0}, \ldots, i_{t}\right\}$ where $i_{j}$ is the row index marked in the box $B_{j}$. Although $I(H)$ depends on the chosen coordinates and on the matrix, if $H^{\prime}$ is another $\mathrm{H}-\mathrm{N}$ matrix for the given sequence, $I\left(H^{\prime}\right)$ may be obtained from $I(H)$ by a permutation of $\{1, \ldots, N\}$; see [Castellanos 1986].

The number of different marked rows is an invariant of the curve in the sense that it does not depend on the chosen embedding, and is called the Arf dimension; see [Castellanos 1986].

Remark 1.1. Since, by Remark 2.2, only a finite number of INPs are necessary to describe the curve, we can give the curves by means of H-N matrices with a finite number of columns, and we suppose that the rest of the columns are composed of zeros.

Remark 1.2. An $\mathrm{H}-\mathrm{N}$ matrix associated with the quadratic transform $C_{i}$, that is, with the sequence $\left\{P_{i-1}, P_{i}, \ldots\right\}$, may be obtained from any H-N matrix associated with the total sequence by considering the $\mathrm{H}-\mathrm{N}$ matrix composed from the columns $i, i+1, \ldots$, and changing in the $i$-th column the entry in the marked row to ane (if it is a zero).

The satellitism order os $\left(P_{i}\right)$ of $P_{i}$ is the number of strict transforms $E_{j}^{(i)}$ of the exceptional divisors $E_{j}$, for $j \leq i$, by the sequence of blowing up at $V_{i}$, containing $P_{i}$. We say that $P_{i}$ is free if $\operatorname{os}\left(P_{i}\right)=1$.

We say that $P_{j}$ is proximate to $P_{i}$, written $P_{j} \rightarrow P_{i}$, if $P_{j}$ belongs to the strict transform $E_{i}^{(j)}$ of the exceptional divisor $E_{i}$. Every point in $E_{i}$ is always proximate to $P_{i-1}$. If $\operatorname{dim}(V) \geq 3$, then $P_{j} \rightarrow P_{i}$ if and only if $j \geq i$ and $E_{i}^{(j+1)} \cap E_{j+1} \neq \varnothing$. Then os $\left(P_{i}\right)=r$ implies that $P_{i}$ is proximate to $r$ points.

Notice that the satellitism order of one point is at most $N$ and that point is proximate to at most $N$ points.

The proximity relations and satellitism orders of a point can be determined in any H-N matrix associated with the curve and they depend only on whether some entries are zero or not.

Proposition 1.3. Let $\left\{P, P_{1}, \ldots, P_{s}, \ldots\right\}$ be a sequence of INPs and $H$ a $H-N$ matrix associated with them. Then

(i) $P_{j}$ is proximate to $P_{i}$, for $j>i$, if $i+1$ is the last index of one box and the marked row in that box has zeros as entries after that box until the $j$-th column.

(ii) The satellitism order $\operatorname{os}\left(P_{j}\right)$ coincides with the number of marked rows having zeros from the last entry 1 in the marked row until the $j$-th column.

Proof. Let $l$ be the marked row index in the box where $j$ is placed at the last column (the index of the chart used in that box). Then $E_{j}^{(j)}$, the strict transform in $P_{j}$ of 
the exceptional divisor $E_{i}$, can be represented by the equation $X_{l}^{(j-i)}=0$. Then the results follow from the construction of the H-N matrix.

Since the chain of INPs determines the curve, all the invariants that we can consider for $C$ are also determined by means of this sequence. Some of them are easily read from the associated H-N matrix (see [Castellanos 1995]) as the multiplicity sequence of $C$, which depends on the proximity relations: $n_{q}$ is associated with $P_{q}$ in the sense that

$$
n_{q}=n_{q+1}+n_{q+2}+\cdots+n_{q+i}
$$

if the $P_{q+l}$, for $l \leq i$, are the only proximate points to $P_{q}$. That is, in the marked row at $P_{q}$ there are zeros in the columns $q+1, q+2, \ldots, q+i$, followed by a nonzero entry. Then $n_{q}$ does not depend on particular values of the entries of the matrix, only on whether or not some of them are zero.

The multiplicity sequence can be written as

$$
E\left(C_{s}\right)=\left\{n, . h ., n, n_{1}, . h_{1} ., n_{1}, \ldots, n_{r-1}, . h_{r-1}, n_{r-1}, 1, . h_{r} ., 1\right\},
$$

where the $h_{i}$ are the length of the boxes of any H-N matrix, and

$$
n_{i}=h_{i+1} n_{i+1}+\cdots+h_{j-1} n_{j-1}+b_{j} n_{j}
$$

if the point corresponding to the penultimate column of the box $B_{i}$ is such that all of its successive points in the sequences, until the one given by the $\left(b_{j-1}\right)$-th column in the box $B_{j}$, are all of the points proximate to it.

\section{Semigroup of values for curve singularities}

The semigroup of values of a curve singularity $C$ is defined to be

$$
\Gamma=\Gamma(C)=\left\{o_{t}(z(t)): z \in R\right\} \subset \mathbb{Z}_{+} .
$$

Since the conductor ideal of $C$ is $\left(t^{c}\right) k \llbracket t \rrbracket$, the semigroup of values of $C$ is a numerical semigroup, that is, it has a conductor $c$, with the property that each positive integer $m \geq c$ lies in $\Gamma$.

Proposition 2.1. Consider a curve singularity $C$ given by the parametrization $\left\{x_{1}(t), \ldots, x_{N}(t)\right\} \subset k \llbracket t \rrbracket$, whose semigroup has conductor $c$. Any parametrization $\left\{y_{1}(t), \ldots, y_{N}(t)\right\} \subset k \llbracket t \rrbracket$ such that $y_{i}(t) \equiv x_{i}(t)\left(\bmod t^{c}\right)$ for $i=1, \ldots, N$ provides the same curve $C$.

Proof. It is sufficient to consider the curve $C^{*}$ given by the parametrization

$$
\left\{\bar{x}_{1}(t), \ldots, \bar{x}_{N}(t)\right\} \subset k \llbracket t \rrbracket,
$$

where $\bar{x}_{i}(t)$ is obtained from $x_{i}(t)$ by truncation of the series mod $t^{c}$. The conductor of the curve generated by this parametrization is also $c$, so for each $m \geq c$ and 
$i \in\{1, \ldots, N\}$ there exists $f_{i}^{(m)}\left(X_{1}, \ldots, X_{N}\right) \in k \llbracket X_{1}, \ldots, X_{N} \rrbracket$, such that

$$
x_{i}(t)-f_{i}^{(c)}\left(x_{1}(t), \ldots, x_{N}(t)\right)+f_{i}^{(c+1)}\left(x_{1}(t), \ldots, x_{N}(t)\right)+\cdots=\bar{x}_{i}(t)
$$

since the rings $R$ and $R^{*}$ of the branches $C$ and $C^{*}$ are complete. So

$$
R^{*}=k \llbracket \bar{x}_{1}(t), \ldots, \bar{x}_{N}(t) \rrbracket \subset R=k \llbracket x_{1}(t), \ldots, x_{N}(t) \rrbracket,
$$

and the converse $R \subset R^{*}$ follows by an analogous argument.

This means that we can consider a finite number of coefficients in the series of any parametrization, that is, for a parametrization of $C$ it is sufficient to consider polynomials in $t$ with coefficients in $k$ with exponents at most $c$.

Remark 2.2. Since exponents greater than $c$ are unnecessary in the parametrization of $C$, only a finite number of columns in any $\mathrm{H}-\mathrm{N}$ associated with the parametrization need be described, and so only a finite number $\tau<c$ of INPs are necessary to describe the curve.

The conductor $c$ cannot be computed from the exponents of any parametrization, nor is there a formula for it in terms of any set of generators of the semigroup. But we can find a bound for $c$ obtained from the multiplicity sequence, that is, from any $\mathrm{H}-\mathrm{N}$ matrix of the curve.

We consider the invariant $\delta$ in the function of the successive blowing ups

$$
\delta(C)=\operatorname{dim}_{k} \bar{R} / R=\operatorname{dim}_{k} R^{(1)} / R+\operatorname{dim}_{k} R^{(2)} / R^{(1)}+\cdots+\operatorname{dim}_{k} R^{(r)} / R^{(r-1)} .
$$

An upper bound for $\operatorname{dim}_{k} R^{(1)} / R$ is given by Elias [1990, Theorem 1.6], as

$$
\frac{1}{2} n(n-1)-\frac{1}{2}(b-1)(b-2),
$$

with $n$ being the multiplicity of $C$ and $b$ being embdim $(C)$.

Then

$$
\delta(C) \leq \frac{1}{2} n(n-1)+\frac{1}{2} n_{1}\left(n_{1}-1\right)+\cdots+\frac{1}{2} n_{r-1}\left(n_{r-1}-1\right)
$$

is an upper bound for $\delta(C)$.

From the definition we can see that $\delta(C)$ is the number of gaps of the semigroup $S(C)$, that is, the set of integers less than the conductor $c$, which do not belong to the semigroup. Also, $2 \delta(C) \geq c$, and so

$$
c^{*}=n(n-1)+n_{1}\left(n_{1}-1\right)+\cdots+n_{r-1}\left(n_{r-1}-1\right)
$$

is an upper bound for the conductor in terms of multiplicity sequence. For plane curves, the conductor coincides with the bound.

We consider the unique minimal system $\left\{\gamma_{0}=n, \gamma_{1}, \ldots, \gamma_{g}\right\} \subset \Gamma$ of generators of $\Gamma$, where

$$
\gamma_{i}=\min \left\{m \in \Gamma: 0<m \notin\left\langle\gamma_{0}, \ldots, \gamma_{i-1}\right\rangle\right\}
$$


with $n$ being the multiplicity of $C$, and where $\langle\ldots\rangle$ denotes the generation of a semigroup. From the definition, for every $i$,

$$
\gamma_{i} \leq c+n-1 \text {. }
$$

The semigroups of plane curves have special properties. Given a plane curve singularity $C$ the semigroup $\Gamma(C)$ satisfies:

(i) $\Gamma(C)$ is symmetric, that is, for each $m<c$, we have $m$ or $m-c \in \Gamma(C)$.

(ii) The minimal generating set $\left\{\gamma_{0}, \ldots, \gamma_{g}\right\}$ satisfies the relations

$$
k_{i} \gamma_{i} \in\left\langle\gamma_{0}, \ldots, \gamma_{i-1}\right\rangle, \quad k_{i} \gamma_{i}<\gamma_{i+1}, \quad \text { and } k_{i}>1
$$

where $d_{i}=\operatorname{gcd}\left(\gamma_{0}, \ldots, \gamma_{i}\right)$ and $d_{i-1}=k_{i} d_{i}$.

Teissier [1973] proves that property (ii) characterizes the numerical semigroups $\Gamma$ "belonging" to a plane curve, that is, with the property that there exists a plane curve $C$ having $\Gamma$ as its semigroup of values.

It is not known whether similar results hold for nonplane curves. We know that any numerical semigroup $\Gamma=\left\langle\gamma_{0}, \ldots, \gamma_{g}\right\rangle$ has an associated monomial curve

$$
C_{\Gamma} \equiv\left\{t^{\gamma_{0}}, \ldots, t^{\gamma_{g}}\right\}
$$

One first question is whether, given a numerical semigroup $\Gamma$, we can find a nonmonomial curve with embedding dimension at most $g+1$ which has $\Gamma$ as its semigroup, and also if we can know the minimum embedding dimension such that there exists a curve with semigroup $\Gamma$.

Example 2.3. A curve containing the semigroup

$\langle 9,10,12\rangle=\left\{9,10,12,18,19,20,21,22,24,27,28,29,30,31,32,34,36+\mathbb{Z}_{+}\right\}$

has the form

$$
\left\{x_{1}=t^{9}+a_{1} t^{9+1}+\cdots, x_{2}=t^{10}+b_{1} t^{10+1}+\cdots, x_{3}=t^{12}+c_{1} t^{12+1}+\cdots\right\} .
$$

We want to find if there exist curves having semigroup $\langle 9,10,12, m\rangle$, with $m>18$ and $m \notin\langle 9,10,12\rangle$, that is, where $m=23,25,26,33,35$. We have to determine the possible exponents in the parametrization, that is, the values of $a_{i}, b_{i}, c_{i}$.

The minimal relation among $9,10,12$ is $2 \times 9+12=3 \times 10$, so the semigroups $\langle 9,10,12, m\rangle$ for $m=23,25,26$ are not possible for a curve with embedding dimension 3. But for the other values we have:

$\langle 9,10,12,33\rangle$ belongs to the curve $\left\{x_{1}=t^{9}, x_{2}=t^{10}+t^{13}, x_{3}=t^{12}\right\}$,

$\langle 9,10,12,35\rangle$ belongs to the curve $\left\{x_{1}=t^{9}, x_{2}=t^{10}, x_{3}=t^{12}+t^{17}\right\}$. 
In this line, we have the following questions for semigroups $\Gamma_{i}$ belonging to curves $C_{i}$ with embedding dimensions $i$, that is, such that $\Gamma\left(C_{i}\right)=\Gamma_{i}$.

Problem 2.4. (i) For each $i>2$ find relations among the generators of the semigroups belonging to a curve which has embedding dimension $i$.

(ii) Given a numerical semigroup $\Gamma$ determine if there exist relations among its elements which determine the minimal embedding dimension for all the curves with semigroup $\Gamma$.

(iii) Determine if the relations found in (i) for any $i$ characterize the semigroups belonging to curves with embedding dimension $i$.

The semigroups of plane curves can be computed by means of the characteristic exponents (some special exponents of the parametrization for zero characteristic of $k$ ) of a Puiseux parametrization, or the multiplicity sequence; see [Zariski 1965]. In the space case $N>2$ it is well known that this is not true.

Example 2.5. The curves

$$
\begin{aligned}
& C_{1} \equiv\left\{x_{1}=t^{6}, x_{2}=t^{8}+t^{11}, x_{3}=t^{10}+t^{13}\right\}, \\
& C_{2} \equiv\left\{x_{1}=t^{6}, x_{2}=t^{8}+t^{11}, x_{3}=t^{10}+2 t^{13}\right\}
\end{aligned}
$$

have the same exponents but their semigroups are given by the generating sets $\Gamma_{1}=\langle 6,8,10,19,21,23\rangle$ and $\Gamma_{2}=\langle 6,8,10,21,23\rangle$. The relation $x_{2}^{2}-x_{1} x_{3}$ gives 19 in the first case, and 22 in the second.

Example 2.6. The curves

$$
\begin{aligned}
& C_{1} \equiv\left\{x_{1}=t^{4}, x_{2}=t^{5}, x_{3}=t^{6}\right\}, \\
& C_{2} \equiv\left\{x_{1}=t^{4}, x_{2}=t^{5}, x_{3}=t^{7}\right\}
\end{aligned}
$$

with $\mathrm{H}-\mathrm{N}$ matrices

$$
H_{1}=\left(\begin{array}{l|llll}
\mathbf{1} & 0 & 0 & 0 & 1 \\
0 & \mathbf{1} & \mathbf{0} & \mathbf{0} & \mathbf{0} \\
0 & 0 & 1 & 0 & 0
\end{array}\right) \quad \text { and } \quad H_{2}=\left(\begin{array}{l|llll}
\mathbf{1} & 0 & 0 & 0 & 1 \\
0 & \mathbf{1} & \mathbf{0} & \mathbf{0} & \mathbf{0} \\
0 & 0 & 0 & 1 & 0
\end{array}\right)
$$

have the same multiplicity sequence $\{4,1\}$, that is, the same proximity relations, but their semigroups are generated by $\Gamma_{1}=\langle 4,5,6\rangle$ and $\Gamma_{2}=\langle 4,5,7\rangle$.

For the plane case, the resolution of the singularity provides exceptional divisors which are $\mathbb{P}^{1} \mathrm{~s}$. Then there are only two possibilities for the position of the infinitely near points, to be the intersection point of two of these $\mathbb{P}^{1} \mathrm{~s}$ (that is, to be satellites) or to be only in one (that is, to be free). In this case, the satellite points determine the multiplicity sequence and the semigroup. For the space case $N>2$ the exceptional divisors are $\mathbb{P}^{N-1} \mathrm{~s}$ or blowing ups of $\mathbb{P}^{N-1} \mathrm{~s}$ and the positions of the INPs have many more possibilities. 
For instance, in Example 2.6, in the first exceptional divisor $E_{1}$ the successive blowing ups (following the points) of the line $\left\{X_{1}=0\right\} \subset E_{1}$ only pass by $P_{1}, P_{2}$ in $C_{1}$, and by $P_{1}, P_{2}, P_{3}$ in $C_{2}$. In this case, this fact together with the proximity relations determine the semigroup. In Section 3 we study the cases when general linear varieties pass by the INPs.

In fact the semigroup also depends on some free points as we see in the following example.

Example 2.7. Consider the curves

$$
\begin{aligned}
& C_{1} \equiv\left\{x_{1}=t^{6}, x_{2}=t^{8}, x_{3}=t^{10}+t^{13}\right\}, \\
& C_{2} \equiv\left\{x_{1}=t^{6}+t^{9}, x_{2}=t^{8}+t^{11}, x_{3}=t^{10}+2 t^{13}+t^{16}\right\},
\end{aligned}
$$

with $\mathrm{H}-\mathrm{N}$ matrices

$$
H_{i}=\left(\begin{array}{c|ccc|ccc}
\mathbf{1} & 0 & 0 & 1 & 0 & 0 & a \\
0 & \mathbf{1} & \mathbf{0} & \mathbf{0} & 0 & 1 & 0 \\
0 & 0 & 1 & 0 & \mathbf{1} & \mathbf{0} & \mathbf{0}
\end{array}\right)
$$

where $a=0$ for $H_{1}$ and $a=1$ for $H_{2}$, with semigroups $\Gamma_{1}=\langle 6,8,10,19,21,23\rangle$ and $\Gamma_{2}=\langle 6,8,10,19,23\rangle$, since $x_{1}^{3}-x_{2} x_{3}$ has value 21 for the first case and 27 in the second one. The points $\left\{P, P_{1}, \ldots, P_{7}\right\}$ are shared and $P_{8}$ is free and it is different in the two cases.

The above and the next examples prove that the semigroup depends on the values of some entries of the H-N matrix, not only on the fact that some entries will be zeros or not.

Example 2.8. Consider the curves

$$
\begin{aligned}
& C_{1} \equiv\left\{x_{1}=t^{20}, x_{2}=t^{21}, x_{3}=t^{24}+t^{27}+t^{29}, x_{4}=t^{26}+t^{29}\right\}, \\
& C_{2} \equiv\left\{x_{1}=t^{20}, x_{2}=t^{21}, x_{3}=t^{24}+2 t^{27}+t^{29}, x_{4}=t^{26}+3 t^{29}\right\},
\end{aligned}
$$

with 21-column H-N matrices

$$
H_{i}=\left(\begin{array}{c|cccccccccccc}
\mathbf{1} & 0 & 0 & 0 & 0 & 0 & 0 & 0 & 0 & 0 & 0 & \ldots & 1 \\
0 & \mathbf{1} & \mathbf{0} & \mathbf{0} & \mathbf{0} & \mathbf{0} & \mathbf{0} & \mathbf{0} & \mathbf{0} & \mathbf{0} & \mathbf{0} & \ldots & \mathbf{0} \\
0 & 0 & 0 & 0 & 1 & 0 & 0 & a & 0 & 1 & 0 & \ldots & 0 \\
0 & 0 & 0 & 0 & 0 & 0 & 1 & 0 & 0 & b & 0 & \ldots & 0
\end{array}\right)
$$

where $a=b=1$ for $C_{1}$, and $a=2$ and $b=3$ for $C_{2}$, with semigroups $\Gamma_{1}=$ $\langle 20,21,24,26,75\rangle$ and $\Gamma_{2}=\langle 20,21,24,26,77\rangle$, since $x_{3}^{3}-x_{1} x_{4}^{2}$ has value 75 for the first case and 77 for the second one.

The next example shows that the semigroup of a curve does not determine the semigroup of the successive blowing ups, as in the plane case. 
Example 2.9. Consider the curves

$$
C_{1} \equiv\left\{x_{1}=t^{6}, x_{2}=t^{8}+t^{9}, x_{3}=t^{27}\right\}, \quad C_{2} \equiv\left\{x_{1}=t^{6}, x_{2}=t^{8}+t^{11}, x_{3}=t^{25}\right\},
$$

with semigroups $\Gamma_{1}=\Gamma_{2}=\langle 6,8,25,27\rangle$. The blowing ups are

$$
\left(C_{1}\right)^{\prime}=\left\{x_{1}=t^{6}, x_{2}=t^{2}+t^{3}, x_{4}=t^{21}\right\}, \quad\left(C_{2}\right)^{\prime}=\left\{x_{1}=t^{6}, x_{2}=t^{2}+t^{5}, x_{3}=t^{19}\right\},
$$

with semigroups $\Gamma_{1}^{\prime}=\langle 2,7\rangle$ and $\Gamma_{2}^{\prime}=\langle 2,9\rangle$.

We now describe two easily computed semigroups associated with any curve $C$. We can consider them as two bounds of $\Gamma(C)$.

First, a curve $C^{*}$ is $A r f$ if its semigroup is determined by its multiplicity sequence $\left\{n, n_{1}, n_{2}, \ldots, n_{r-1}, n_{r}=1\right\}$ and it has the form

$$
\Gamma\left(C^{*}\right)=\left\{n, n+n_{1}, n+n_{1}+n_{2}, \ldots, n+n_{1}+\cdots+n_{r-1}+1+\mathbb{N}\right\} .
$$

The Arf closure $C^{*}=\operatorname{Spec}\left(R^{*}\right)$ of a curve $C$ is the minimum Arf curve such that $R \subset R^{*}$. (See [Lipman 1971] for details.) This curve has the same multiplicity sequence as $C$ and its semigroup is the one determined by the multiplicity sequence as above.

This gives us the first upper bound

$$
\Gamma(C) \subset \Gamma\left(C^{*}\right) .
$$

Second, let $k$ be a field with characteristic zero, then any curve $C$ has a Puiseux parametrization, that is, a parametrization with the form

$$
\left\{x_{1}=t^{n}, x_{2}=a_{n, 2} t^{n}+a_{n+1,2} t^{n+1}+\cdots, \ldots, x_{N}=a_{n, N} t^{n}+a_{n+1, N} t^{n+1}+\cdots\right\},
$$

where $n$ is the multiplicity of $C$. We can define the characteristic sequence associated to a Puiseux parametrization in a similar way to plane curves. We reorder the exponents of the series $x_{2}(t), \ldots, x_{N}(t)$ in an increasing chain as $\left\{n, \alpha_{1}, \alpha_{2}, \ldots\right\}$. We set

$\beta_{0}=n$,

$\beta_{1}=$ the minimum $\alpha_{j_{1}}$ such that $\operatorname{gcd}\left(n, \alpha_{j_{1}}\right)<n$,

$\beta_{i}=$ the minimum $\alpha_{j_{i}}$ such that $\operatorname{gcd}\left(n, \beta_{1}, \ldots, \beta_{i-1}, \alpha_{i}\right)<\operatorname{gcd}\left(n, \beta_{1}, \ldots, \beta_{i-1}\right)$

to obtain characteristic exponents $\left\{n, \beta_{1}, \ldots, \beta_{u}\right\}$, with $\operatorname{gcd}\left(n, \beta_{1}, \ldots, \beta_{u}\right)=1$.

Remark 2.10. These exponents are invariants of the curve $C$, since they are the characteristic exponents of the generic plane projection curve $C_{\pi}$, obtained by the parametrization

$$
\left.\left\{x_{1}(t), y_{2}(t)\right\}=\lambda_{2} x_{2}(t)+\cdots+\lambda_{N} x_{N}(t)\right\}
$$

in the field $K=\overline{k\left(\lambda_{2}, \ldots, \lambda_{N}\right)}$ (the algebraic closure of $k\left(\lambda_{2}, \ldots, \lambda_{N}\right)$ ). 
Then from the exponents of a Puiseux parametrization we get the generators of the semigroup of the generic plane projection, which gives us the second bound

$$
\Gamma\left(C_{\pi}\right) \subset \Gamma(C) .
$$

\section{Linear proximity of dimension $l$}

We now consider a different notion of proximity, generalizing that in the case of plane curves. It is a generalization of that given by [Campillo et al. 1996] for the monomial case, and by [Piltant 1997; Castellanos 2002] for the general case.

Definition 3.1. Given a sequence $\left\{P, P_{1}, \ldots, P_{s}\right\}$ of INPs, we say that $P_{i}$, for $1 \leq i \leq s$, is linearly proximate to $P$ of dimension $l$, or $l$-linearly proximate to $P$, if $P_{i}$ is proximate to $P$ and there is a linear $l$-dimensional subvariety $F_{l}$ of the first exceptional divisor $E_{1}$, with $P_{1} \in F_{l}$, such that its strict transforms $F_{l}^{(j)}$ along the chain of blowing ups contain $P_{j}$, and there is no such linear subvariety with dimension less than $l$.

For $l=1$ the variety is a line, and this definition coincides with that of linear proximity given in [Campillo et al. 1996; Piltant 1997; Castellanos 2002].

Given a sequence $\left\{P, P_{1}, \ldots, P_{s}\right\}$ of INPs, consider the points $P_{j}$ proximate to $P$. For $l \geq 2$, let $P_{k_{l}}$ be the first point in the sequence which is $l$-linearly proximate to $P$ (so that $P_{k_{l}-1}$ is only $(l-1)$-linearly proximate to $P$ ). We can thus consider the unique sequence of indices $k_{2}, \ldots, k_{r}$, where $r \leq N-1$.

Definition 3.2. We call the indices $k_{2}, \ldots, k_{r}$ just described the linear proximity invariants of the sequence $\left\{P, P_{1}, \ldots, P_{s}\right\}$ of INPs.

We can compute the type of linear proximity of the points, and thus the linear proximity invariants themselves, with any $\mathrm{H}-\mathrm{N}$ matrix associated to the sequence of INPs.

Proposition 3.3. Consider a sequence $\left\{P, P_{1}, \ldots, P_{s}\right\}$ of INPs in $R$ where, for $m \leq s,\left\{P_{1}, \ldots, P_{m}\right\}$ are the points proximate to $P$, and suppose $k_{2}, \ldots, k_{r}$ are the linear proximity invariants of this sequence. Then the sequence has an H-N matrix $H$ consisting of

(i) a first marked row whose first $(m-1)$ entries are zero,

(ii) a row where the first entry following a marked row is zero,

(iii) $(r-1)$ rows whose first $\left(k_{i}-1\right)$ entries are zero, for $i \in\{2, \ldots, r\}$, at least one of which has a nonzero $\left(k_{i}+1\right)$-th entry, and

(iv) $(N-r-1)$ rows whose first $(m-1)$ entries are zero.

Proof. Suppose the marked rows in boxes $B_{0}$ and $B_{1}$ are, respectively, the first and the second ones. Since $P_{1}, \ldots, P_{m}$ are proximate to $P$, then $B_{0}$ has only one 
column $\left(\mathbf{1}, a_{2}, \ldots, a_{N}\right)^{t}$, and the first row of the matrix has zero entries from after the marked row until the $m$-th column. Let $\left\{x_{1}, \ldots, x_{N}\right\}$ be a parameter system for $\mathfrak{m} \subset R$, then by setting $x_{1}=x_{1}^{\prime}$ and $x_{j}=x_{j}^{\prime}+a_{j} x_{1}$ for $2 \leq j \leq N$, we transform the first column into $(1,0, \ldots, 0)^{t}$, and by setting $x_{1}^{\prime}=x_{1}^{\prime \prime}$, setting $x_{2}^{\prime}=x_{2}^{\prime \prime}$, and $x_{j}^{\prime}=x_{j}^{\prime \prime}+a_{j 1}^{1} x_{2}^{\prime}$ for $3 \leq j \leq N$, the second column becomes $(0, \mathbf{1}, 0, \ldots, 0)^{t}$. Since $P_{1}$ and $P_{2}$ are 1-linearly proximate to $P$, the line $F_{1}$ (from Definition 3.1) has equations $x_{3}=x_{4}=\cdots=x_{N}=0$, and then for $P_{3}, \ldots, P_{k_{2}}$, the columns are $(0, \mathbf{0}, 0,0, \ldots, 0)^{t}$, so $k_{2} \leq h_{1}+1$ and there exists a nonzero $a_{j\left(k_{2}+1\right)}^{1}$ or $a_{j 1}^{1}=$ $a_{j 2}^{1}=\cdots=a_{j h_{1}}^{1}=0$ and the marked row in $R_{h_{1}+2}$ is the $i$-th, for $3 \leq i \leq N$. Set $j=3$ and the equation for the 2-linear variety is $x_{4}=x_{5}=\cdots=x_{N}=0$.

Now suppose we have the result for $k_{i-1}$, that is, suppose the $(i+j)$-th rows, for $0 \leq j \leq N-i$, have zero entries before column $k_{i}$ and a nonzero entry at least in column $k_{i}$, and set the index of the row of that entry to be $i+1$. Let $x_{1}=x_{1}^{\prime}, \ldots, x_{i+1}=x_{i+1}^{\prime}$ and $x_{u}=x_{u}^{\prime}+\left(a_{u l}^{j} / a_{(i+1) l}^{j}\right) x_{i+1}$ for $i+2 \leq u \leq N$ and suppose that the $l$-th column corresponding to $P_{k_{i}}$ is in box $B_{j}$. Then the equations for the $i$-linear variety $F_{i}$ are $x_{i+2}=\cdots=x_{N}=0$, and rows $i+2, \ldots, N+1$ all have zero entries before column $k_{i+1}$.

Corollary 3.4. Consider a sequence $\left\{P, P_{1}, \ldots, P_{s}\right\}$ in $R$ such that the points $\left\{P_{1}, \ldots, P_{m}\right\}$, where $m \leq s$, are proximate to $P$ and have satellitism order 2 .

(i) If $P_{i}, \ldots, P_{m}$ are l-linearly proximate to $P$ then any $H$ - $N$ matrix $H_{i}$ associated to the points $\left\{P, P_{1}, \ldots, P_{i}\right\}$, has rank $l+1$.

(ii) If there is a rank-(l+1)H-N matrix $H_{i}$ for the INPs $\left\{P, P_{1}, \ldots, P_{i}\right\}$, where $1 \leq i \leq m$, then $P_{i}$ is l-linearly proximate to $P$.

Proof. If $P_{i}$ is $l$-linearly proximate to $P$ then, by Proposition 3.3 , given any $\mathrm{H}-\mathrm{N}$ matrix for the points $\left\{P, P_{1}, \ldots, P_{i}\right\}$ we can construct a rank- $(l+1) \mathrm{H}-\mathrm{N}$ matrix $H_{i}^{*}$, thus $H_{i}$ also has rank $(l+1)$.

Suppose that part (ii) holds for $P_{i-1}$ and consider a rank- $(l+1) \mathrm{H}-\mathrm{N}$ matrix $H_{i}$ for $\left\{P, P_{1}, \ldots, P_{i}\right\}$. Set $H_{i-1}$ to be the $\mathrm{H}-\mathrm{N}$ matrix obtained with the first $(i-1)$ columns of $H_{i}$. Then we make $H_{i-1}$ a triangular matrix by modifications on the rows in a similar manner to the proof of Proposition 3.3. Then if $H_{i-1}$ has rank $(l+1)$, we can suppose that the equations of an $l$-linear variety $F_{l}$ of $E_{1}$ passing through $P_{1}, \ldots, P_{i-1}$ are $x_{l+2}=\cdots=x_{N}=0$. Then with the same modifications on $H_{i}$ we see that the same variety $F_{i}$ also passes through $P_{i}$, and $P_{i}$ is $l$-linearly proximate to $P$. In the case that $H_{i-1}$ has rank $l$, applying the same process to $H_{i-1}$, shows that the equations of an $(l-1)$-linear variety $F_{l-1}$ of $E_{1}$ passing through $P_{1}, \ldots, P_{i-1}$ are $x_{l+1}=\cdots=x_{N}=0$. Applying the same modifications to $H_{i}$, since $H_{i}$ has rank $l+1$, we see that the new matrix $H_{i}^{*}$ must have a nonzero entry in the $i$-th column of rows $(l+1), \ldots, N$, and so $P_{i}$ is $l$-linearly proximate to $P$. 
From the above discussion, we can compute some elements of the semigroup of a curve by means of the linear proximity invariants.

Proposition 3.5. The linear proximity of all the dimensions provides some elements of the semigroup of a curve and the semigroup of its successive blowing ups.

Proof. We prove that the linear proximity invariants associated with any sequence of INPs can be read in the semigroup of values of the curve.

Consider the subset

$$
\Gamma_{u}\left(C_{s}\right)=v\left(\mathfrak{m} \backslash \mathfrak{m}^{2}\right)=\left\{o_{t}\left(\lambda_{1} x_{1}(t)+\cdots+\lambda_{N} x_{N}(t)\right)<2 n, \lambda_{i} \in k\right\}
$$

of the semigroup $\Gamma\left(C_{s}\right)$, where $\left.\left\{x_{1}(t), \ldots, x_{N}(t)\right)\right\}$ is a parametrization of the curve. Let $\left\{n, \gamma_{1}, \ldots, \gamma_{g}\right\}$ be the minimal set of generators of $\Gamma(C)$, and suppose that $\left\{n, \gamma_{1}, \ldots, \gamma_{u}\right\}$ are all the generators with $\gamma_{i}<2 n$. The multiplicity sequence of $C$ is

$$
E(C)=\left\{n, n_{1}, . h_{1} ., n_{1}, \ldots, n_{r-1}, . h_{r-1}, n_{r-1}, 1, . h_{r} ., 1\right\}
$$

and from (1-2) we suppose that $n=h_{1} n_{1}+\cdots+h_{l-1} n_{l-1}+b_{l} n_{l}$. Then $\gamma_{1}=h n+n_{1}$, and by looking at any H-N matrix we can see that each $\gamma_{j}$, for $j \geq 2$, has an expression

$$
\gamma_{j}=n+h_{1} n_{1}+\cdots+h_{r_{j}-1} n_{r_{j}-1}+q_{j} n_{r_{j}},
$$

and since $\gamma_{j}<2 n$, either $j<l$, or $j=l$, in which case $q_{l} \leq b_{l}$. Then the linear proximity invariants $k_{j}$ are

$$
k_{j}=1+h_{1}+\cdots+h_{r_{j}}+q_{j}
$$

since there is a parametrization $\left.\left\{y_{1}(t), \ldots, y_{N}(t)\right)\right\}$, with $y_{j}=\lambda_{j 1} x_{1}+\cdots+\lambda_{j N} x_{N}$, such that $o_{t}\left(y_{1}(t)\right)=n$ and $o_{t}\left(y_{j}(t)\right)=\gamma_{j-1}$ for $j=2, \ldots, u+1$, and for any H-N matrix associated to $\left.\left\{y_{1}(t), \ldots, y_{N}(t)\right)\right\}$ the $(j+1)$-th row, for $2<j \leq u$, has zero entries in the first $k_{j}$ columns, and at least one such row has a nonzero entry in column $k_{j}+1$.

For the $i$-th blowing up of $C$, its $\mathrm{H}-\mathrm{N}$ matrix $C^{(i)}$ occurs as a submatrix of any H-N matrix of $C$; see Remark 1.2. Then, as above, some elements of the semigroup of the blowing up $C^{(i)}$ are described by the linear proximate invariants.

Example 3.6. (i) Consider the curves in Example 2.6. For $C_{1}$, the points $P_{1}$ and $P_{2}$ are the only ones 1 -linearly proximate to $P$. For $C_{2}$, the point $P_{3}$ is also 1-linearly proximate to $P$.

(ii) Consider the curves in Example 2.8. For both curves $P_{1}, P_{2}, P_{3}, P_{4}$, are the only points 1-linearly proximate to $P$, and $P_{5}$ and $P_{6}$ are 2-linearly proximate to $P$.

We consider now an example with several boxes in its H-N matrix. 
Example 3.7. Consider the curves

$$
\begin{gathered}
C_{1} \equiv\left\{t^{27}+t^{26}+t^{25}+t^{24}, t^{40}+2 t^{38}+2 t^{39}+2 t^{37}+t^{36}, t^{46}+2 t^{44}+2 t^{45}+2 t^{43}+t^{42}\right\}, \\
C_{2} \equiv\left\{t^{28}+t^{27}+t^{25}+t^{24}, t^{41}+t^{38}+2 t^{40}+2 t^{37}+t^{39}+t^{36},\right. \\
\left.t^{47}+t^{44}+2 t^{46}+2 t^{43}+t^{45}+t^{42}\right\},
\end{gathered}
$$

with $\mathrm{H}-\mathrm{N}$ matrices

$$
H_{i}=\left(\begin{array}{l|l|ll|llllll}
\mathbf{1} & 0 & 0 & 1 & 0 & a & b & 0 & 0 & 0 \\
0 & \mathbf{1} & 0 & 1 & \mathbf{1} & \mathbf{0} & \mathbf{0} & \mathbf{0} & \mathbf{0} & \mathbf{0} \\
0 & 0 & \mathbf{1} & \mathbf{0} & 0 & 0 & 0 & 0 & 0 & 1
\end{array}\right)
$$

where $a=1$ and $b=0$ for $C_{1}$ with $\Gamma_{1}=\langle 26,38,44,115,209,221,233,239,251\rangle$, and $a=0$ and $b=1$ for $C_{2}$, with $\Gamma_{2}=\langle 24,36,42,73,87,146,190,221,239\rangle$. For $C_{1}$, the points $P_{4}$ and $P_{5}$ are the only ones 1 -linearly proximate to $P_{3}$. For $C_{2}$, the point $P_{6}$ is also 1-linearly proximate to $P_{3}$.

Linear proximity of all dimensions is not enough to describe the semigroups of values, and we must consider proximity given by varieties with degree higher than 1. In Section 4 we consider hypersurfaces passing through points of higher degree.

Example 3.8. Consider the curves

$$
\begin{aligned}
& C_{1} \equiv\left\{x_{1}=t^{10}, x_{2}=t^{11}, x_{3}=t^{12}+t^{14}+t^{16}\right\} \\
& C_{2} \equiv\left\{x_{1}=t^{10}, x_{2}=t^{11}, x_{3}=t^{12}+t^{14}+t^{15}+t^{16}\right\},
\end{aligned}
$$

with H-N matrices

$$
H_{i}=\left(\begin{array}{c|llllllllll}
\mathbf{1} & 0 & 0 & 0 & 0 & 0 & 0 & 0 & 0 & 0 & 1 \\
0 & \mathbf{1} & \mathbf{0} & \mathbf{0} & \mathbf{0} & \mathbf{0} & \mathbf{0} & \mathbf{0} & \mathbf{0} & \mathbf{0} & \mathbf{0} \\
0 & 0 & 1 & 0 & 1 & a & 1 & 0 & 0 & 0 & 0
\end{array}\right)
$$

where $a=0$ for $C_{1}$ and $a=1$ for $C_{2}$, with $\Gamma_{1}=\langle 10,11,12,26\rangle$ and $c_{1}=30$, and $\Gamma_{2}=\langle 10,11,12,25,38\rangle$ and $c_{2}=40$. Both curves have the same proximity and linear proximity, but for $C_{1}$ there exists a conic $X_{1} X_{3}-X_{2}^{2}-X_{3}^{2}$ in the first exceptional divisor passing through $P_{1}, P_{2}, \ldots, P_{6}$, while for $C_{2}$ there are no such conics since a general conic passing through those points would have the form $X_{1} X_{3}-X_{2}^{2}+a_{3} X_{3} X_{2}+a_{4} X_{3}^{2}$, and there are no values for $a_{3}$ or $a_{4}$ compatible with the sequence of blowing ups following the points.

Example 3.9. Consider the curves of Example 2.8. For both curves the proximity and linear proximity are the same. For $C_{2}$ there is a cubic surface in the first exceptional divisor $X_{3}^{3}-X_{1} X_{4}^{2}$ passing through $P_{1}, P_{2}, \ldots, P_{10}$, but for $C_{1}$ there are no such cubic surfaces. This follows from Noether's formula (4-2), since the value of the semigroup has to be reached from $3 \times 20+m_{1}+\cdots$, and it is easy to check that this is not possible with the values of the semigroup $\Gamma_{1}$, since $77 \notin \Gamma_{1}$. 


\section{Hypersurfaces of maximal contact}

We have seen in the above examples that in order to describe the semigroup of a space curve, we have to consider proximity given by hypersurfaces of degree higher than 1. In this section we will consider, from different points of view, how these varieties determine elements of the semigroup.

Given a curve $C \hookrightarrow V$, for any hypersurface $F$ such that $C \nsubseteq F$, given by $F\left(X_{1}, \ldots, X_{N}\right)=0$, the intersection multiplicity $I_{P}(F, C)$ between $F$ and $C$ at $P$ can be computed as

$$
I_{P}(F, C)=o_{t}\left(F\left(x_{1}(t), \ldots, x_{N}(t)\right)\right.
$$

with $\left.\left\{x_{1}(t), \ldots, x_{N}(t)\right)\right\}$ being a parametrization of $C$. This value is an element of the semigroup $\Gamma(C)$.

Let $V=\operatorname{Spec}(A)$ as in Section 1 and consider the sequence $\left\{P, P_{1}, \ldots, P_{s}\right\}$ of INPs associated to the curve $C$, with $s \geq \tau$ (see Remark 2.2), in order to determine completely the curve. We have a sequence

$$
A \hookrightarrow A_{1} \hookrightarrow \cdots \hookrightarrow A_{s} \hookrightarrow A_{v}
$$

of rings, where $A_{v}=\mathrm{O}_{V_{s+1}, \eta}$ and $\eta$ is the generic point of the exceptional divisor $E_{s+1}$ in $V_{s+1}$. Then we have associated to them a divisorial valuation $v$ over the quotient field $K=k(V)$ given by the discrete valuation ring $A_{v}$ (the quadratic prime divisor), that is, $\operatorname{tr} \operatorname{deg}_{k}\left(A_{v} / \mathfrak{m}_{v}\right)+1=\operatorname{tr} \operatorname{deg}_{k} k(V)$, where $\mathfrak{m}_{v}$ is the maximal ideal of $A_{v}$.

Associated to any $A_{i}$ is the corresponding order valuation ord ${ }_{i}$ of $K$ defined, for $0 \neq F \in A_{i}$, by

$$
\operatorname{ord}_{i}(F)=\max \left\{n: F \in \mathfrak{M}_{i}^{n}\right\} .
$$

For $F \in A, F^{(i)}$ is the strict transform of $F$ (that is, we take off the part corresponding to the exceptional divisor) by the chain in $A_{i}$.

So we have the above valuation $v=\operatorname{ord}_{s}$, determined by $C$ associated to the sequence of INPs in the previous section. In fact, $\operatorname{ord}_{s}(F)=I_{P}(F, C)$ for any $F(\underline{X})$; see [Castellanos 2002, Proposition 2.0.1].

We say that a hypersurface $F$ passes exactly by $\left\{P, P_{1}, \ldots, P_{j}\right\}$ if those points are precisely the ones in common with $C$. Then $F^{(j+1)}$ becomes a unit. Set $\left\{m, m_{1}, \ldots, m_{j}\right\}$, where $m=\operatorname{ord}(F)$, and $m_{i}=\operatorname{ord}_{i}(F)$ is the order of any hypersurface $F$ at $P_{i}$.

Proposition 4.1. The semigroup of the curve is determined by means of the set $\left\{m, m_{1}, \ldots, m_{j}\right\}$ of orders, where $m_{i}=\operatorname{ord}_{i}(F)$ denotes the order of any hypersurface $F$ at $P_{i}$ (with $\left\{P, P_{1}, \ldots, P_{s}\right\}$ being the INPs of the curve), and the multiplicity sequence of the curve is $\left\{n, n_{1}, \ldots, n_{r}=1, n_{r+1}=1, \ldots, n_{s}=1\right\}$. 
Proof. The semigroup $\Gamma_{s}=\left\{\operatorname{ord}_{s}(F): F \in \mathfrak{M} \backslash\{0\}\right\}$ associated with the valuation $\operatorname{ord}_{s}$ coincides with the semigroup

$$
\Gamma(C)=\left\{o_{t}\left(F\left(x_{1}(t), \ldots, x_{N}(t)\right)\right): 0 \neq F \in k \llbracket \underline{X} \rrbracket\right\}
$$

of values of the curve $C$. The intersection multiplicity can be computed from the sequence of INPs of $C$ by means of a generalization of Noether's formula [Lipman 1988]

$$
v(F)=v\left(F^{(s)}\right)+\sum_{j=0}^{s-1} \operatorname{ord}_{j}\left(F^{(j)}\right) v\left(\mathfrak{m}_{j}\right),
$$

where $v$ is any valuation associated to the sequence of INPs. In this case the valuation is given by $C$, and $v\left(\mathfrak{m}_{i}\right)$ is the minimum value of the maximal ideal of $A_{i}$, that is, the multiplicity $n_{i}$ of the $i$-th quadratic transform $C_{i}$. Thus, in our case,

$$
\begin{aligned}
I_{P}(F, C) & =\operatorname{ord}(F) n+\operatorname{ord}_{1}\left(F^{(1)}\right) n_{1}+\cdots+\operatorname{ord}_{j}\left(F^{(j)}\right) n_{j} \\
& =m_{1} n_{1}+\cdots+m_{j} n_{j}
\end{aligned}
$$

if $\left\{P, P_{1}, \ldots, P_{j}\right\}$ are the only INPs passed through by $F$.

Remark 4.2. In order to compute the semigroups, we have to determine what sequences $\left\{m, m_{1}, \ldots, m_{s}: m_{i} \geq m_{i+1}\right\}$ of orders are verified by hypersurfaces passing through $\left\{P, P_{1}, \ldots, P_{s}\right\}$. In Proposition 4.10 we see that in some sense this sequence of orders is bounded.

Moreover, we are also interested in determining the sequences of orders of hypersurfaces that provide the minimal sets of generators for the semigroup.

For plane curves these hypersurfaces are curves, and the minimal set of generators is determined by some of them: the so called maximal contact curves of high genus $\Phi$; see [Campillo 1980; Casas-Alvero 2000]. Consider a plane curve $C$ with characteristic exponents $\left\{n, \beta_{1}, \ldots, \beta_{g}\right\}$ (we call $\mathrm{g}$ the genus) and semigroup $\left\langle n, \bar{\beta}_{1}, \ldots, \bar{\beta}_{g}\right\rangle$. We say that the curve $\Phi_{i}$ has maximal contact of genus $i$ if $\Phi_{i}$ has characteristic sequence

$$
\left\{n / e_{i}, \beta_{1} / e_{i}, \ldots, \beta_{i} / e_{i}\right\}
$$

where $e_{i}=\operatorname{gcd}\left(n, \beta_{1}, \ldots, \beta_{i}\right)$, and shares with $C$ the INPs $\left\{P, P_{1}, \ldots, P_{s_{i}}\right\}$ necessary to determine the first $i$ characteristic exponents.

The intersection multiplicity $I_{P}\left(\Phi_{i}, C\right)=\bar{\beta}_{i}$ is, as we have seen, determined by the geometry of the INPs (by the proximity relations). That is, by means of the multiplicity sequence of $C$, and since the sequence $\left\{n^{(i)}, n_{1}^{(i)}, \ldots, n_{s_{i}}^{(i)}=1\right\}$ of orders of $\Phi_{i}$ is the multiplicity sequence of $\Phi_{i}$, (where $m_{j}=n_{j}^{(i)}$ in this case), we have

$$
\bar{\beta}_{i}=n^{(i)} n+n_{1}^{(i)} n_{1}+\cdots+n_{s_{i}}^{(i)} n_{s-i}
$$


Some special maximal contact curves $\Phi_{i}^{*}$ can be seen from a different point of view as the approximate roots of the curve $C$; see [Abhyankar and Moh 1973a; 1973b; Popescu-Pampu 2003; Casas-Alvero 2000].

The $d$-approximate root $d \mid n$ of an irreducible plane curve $C$ given by a Weierstrass polynomial $G\left(x_{1}, x_{2}\right)$ is a Weierstrass polynomial $Q\left(x_{1}, x_{2}\right)$ which satisfies $\operatorname{deg}\left(G-Q^{d}\right)>n-n / d$.

Let $e_{i}=\operatorname{gcd}\left(n, \beta_{1}, \ldots, \beta_{i}\right)$ and let $\left\{x_{1}=t^{n}, x_{2}=\alpha(t)\right\}$ be the Puiseux series of $C$. The $e_{i}$-approximate root of $C$ is the Weierstrass polynomial of an irreducible plane curve which has the same $i$ characteristic exponents $\left\{n / e_{i}, \beta_{1} / e_{i}, \ldots, \beta_{i} / e_{i}\right\}$ as $C$. It determines, as above, a curve $\Phi_{i}^{*}$, whose value $\bar{\beta}_{i}$ is one of the minimal generators of the semigroup; see [Casas-Alvero 2000, 5.9].

Remark 4.3. From Proposition 4.1 for a space curve $C$ we consider the set of hypersurfaces $F_{i}$ providing the minimal set of generators $\left\{n, \gamma_{1}, \ldots, \gamma_{g}\right\}$ of the semigroup, as the approximate roots of $C$. The problem is to characterize these $F_{i}$ by means of the geometry of the INPs.

All the possible sequences $\left\{m, m_{1}, \ldots, m_{j}\right\}$ as above, determine the semigroup of the curve. For plane curves, as noted above, the sequences $\left\{m, m_{1}, \ldots, m_{j}\right\}$ of orders are the multiplicity sequence of some plane curves, so they are characterized by the proximity relations, for plane curves, of the sequence of INPs of the curve, that is, it is enough that

$$
m_{i}=m_{i+1} \text {, or } m_{i}=m_{i+1}+\cdots+m_{j-1}+m_{j} \text { and } m_{i+1}=\cdots=m_{j-1} \geq m_{j} .
$$

For space curves, the sequence of $m_{i}$ follow neither the proximity relations of the INPs nor the $l$-linear proximity relations. For the curve $C_{1}$ in Example 2.8, $X_{3}^{3}-X_{1} X_{4}^{2}$ has the sequence of 16 orders $3,1, \ldots, 1$, the corresponding value is $75=3 \times 20+15$ and the proximity relations of the INPs given by $20=1+\cdots+1$ are not reached by the orders.

In the following we will consider all the hypersurfaces passing through subsequences of the INPs of the curve with prefixed orders, that is clusters of INPs. They provide finitely supported complete ideals.

Consider a curve $C$ and its sequence $\left\{P, P_{1}, \ldots, P_{j} \ldots\right\}$ of INPs. Given an ideal $I \subset A$ we define

$$
\operatorname{ord}_{s} I=\min \left\{\operatorname{ord}_{s}(F): F \in I\right\}
$$

and $\operatorname{ord}_{i} I=\operatorname{ord}_{i} I^{(i)}$, where $I^{(i)}$ is the weak transform of $I$ in $A_{i}$, that is,

$$
I^{(i)}=(x)^{-m_{i-1}} I^{(i-1)} A_{i},
$$

where $m_{i}=\operatorname{ord}_{i} I^{(i)}=\max \left\{n: I^{(i)} \subset\left(\mathfrak{M}_{i}\right)^{n}\right\}\left(\mathfrak{M}_{i}\right.$ being the maximal ideal of $\left.A_{i}\right)$ and $x$ is a generator of the principal ideal $\mathfrak{M}_{i-1} A_{i}$. 
An ideal $I \subset A$ is finitely supported [Lipman 1988] if $I$ is primary for the maximal ideal $\mathfrak{M}$ of $A$, and there are only a finite number of base points. If the base points are a sequence $\left\{P, P_{1}, \ldots, P_{s}\right\}$ of INPs of $P$, we have that $I A_{s}$ is invertible. We say that $\left\{P, P_{1}, \ldots, P_{s}\right\}$ are the base points of $I$.

We set $B(I)=\left\{\operatorname{ord}_{s}\left(I^{(i)}\right)\right\}_{i=0,1, \ldots, s}$. If we consider a sequence $\left\{P, P_{1}, \ldots, P_{r}\right\}$ of INPs with $r>s$, then since $I$ becomes invertible at $P_{s}$, we have $I^{(j)} A_{j}=A_{j}$ for $j>r$, and its order is 0 .

An ideal $I \subset A$ is complete if $I$ contains all elements $x \in A$ satisfying

$$
x^{n}+a_{1} x^{n-1}+\cdots+a_{n}=0
$$

where $a_{j} \in I^{j}$ for $1 \leq j \leq n$, with $I^{j}$ denoting the $j$-th power of $I$. The integral closure $\bar{I}$ of an ideal $I$ is the minimal complete ideal containing $I$.

Given ideals $I, J \subset A$ we have (see [Lipman 1988, 1.9.10]):

(i) $B(I \cdot J)=B(I)+B(J)$.

(ii) $B(I)=B(J) \Longleftrightarrow \bar{I}=\bar{J}$.

Definition 4.4. Given $F \in A$, the virtual transform of $F$ of order $m$ in $P_{1}$ is $\widetilde{F}_{1}=X^{-m} F_{1}$, if $\operatorname{ord}(F) \geq m$, and $F_{1}$ is the total transform of $F$ in $A_{1}$.

We say that the hypersurface $F \in A$ passes through $P, P_{1}, \ldots, P_{j}$ with orders $m, m_{1}, \ldots, m_{j}$ if $\operatorname{ord}(F) \geq m$ and $\widetilde{F}_{1}$, the virtual transform of $F$ in $A_{1}$ of order $m$, has order at least $m_{1}$, and for all $i \leq j$ the virtual transform of $\widetilde{F}_{i-1}$ in $A_{i}$ of order $m_{i-1}$ has order at least $m_{i}$.

Let $I_{m, m_{1}, \ldots, m_{j}}$ be the complete ideal of all the hypersurfaces passing through $P, P_{1}, \ldots, P_{j}$ with orders $m, m_{1}, \ldots, m_{j}$. This ideal cannot be finitely supported, but it passes by only a finite number of INPs of the sequence given by $C$.

Proposition 4.5. Consider the sequence $\mathscr{P}=\left\{P, P_{1}, \ldots, P_{i}, \ldots\right\}$ of INP of $C$. Then the ideal $I_{m, m_{1}, \ldots, m_{j}}$, as defined above, passes through a finite number of INPs $P, P_{1}, \ldots, P_{u}$ (for some $u \geq j$ ) of the sequence $\mathscr{P}$.

Proof. Consider a basis $\left\{G_{1}, \ldots, G_{d}\right\}$ of $I_{m, m_{1}, \ldots, m_{j}}$, and let $G=\lambda_{1} G_{1}+\cdots+\lambda_{d} G_{d}$, (where $\lambda_{1}, \ldots, \lambda_{d}$ are generic), be a generic element of $I_{m, m_{1}, \ldots, m_{j}}$. Then for any sufficiently large subsequence of $\mathscr{P}$, containing the first $j$ INPs, let $v$ be the associated valuation. From the preceding discussion, $v$ coincides with the valuation given by the curve, and if we extend the parametrization for $C$ to the field $k\left(\lambda_{1}, \ldots, \lambda_{d}\right)$, we see that

$$
\infty>v(G)=I_{P}(G, C)=\operatorname{ord}(G) n+\operatorname{ord}_{1}\left(G^{(1)}\right) n_{1}+\cdots+\operatorname{ord}_{u}\left(G^{(u)}\right) n_{u} .
$$

Then the ideal $I_{m, m_{1}, \ldots, m_{j}}$ passes through a finite number $u+1$ of INPs of the sequence $\mathscr{P}$. 
Given a sequence $\left\{P, P_{1}, \ldots, P_{j}\right\}$ of INPs of a curve, and a monotonically increasing sequence $\left\{m, m_{1}, \ldots, m_{j}: m_{i} \geq m_{i+1}\right\}$ of integers, the hypersurfaces passing by the points with those orders may have to pass by more points; see Example 4.8. Then, we can consider the following clusters.

Definition 4.6. The shadow cluster generated by the ideal $I_{m, m_{1}, \ldots, m_{j}}$ is the cluster

$$
S_{m, m_{1}, \ldots, m_{u}},
$$

for $u \geq j$, composed from the subsequence $P, P_{1}, \ldots, P_{u}$ of the sequence $\mathscr{P}$ of INPs of $C$ formed by the base points of $I_{m, m_{1}, \ldots, m_{j}}$ contained in $\mathscr{P}$, the weights being the orders of $\left(I_{m, m_{1}, \ldots, m_{j}}\right)^{\left(P_{i}\right)}$ corresponding to the points $P, P_{1}, \ldots, P_{s}$, that is, $B\left(I_{m, m_{1}, \ldots}\right) \cap \mathscr{P}$.

By Proposition 4.5, the shadow cluster $S_{m, m_{1}, \ldots, m_{u}}$ has a finite number of points and is well defined.

Proposition 4.7. The semigroup of values of a curve $C$ is determined by the shadow clusters for all clusters contained in the sequence of infinitely near points of $C$ starting at $P$.

Proof. Let $\left\{P, P_{1}, \ldots, P_{s}, \ldots\right\}$ be the INPs of $C$. The values of $\Gamma(C)$ can be obtained by Noether's formula (4-2), applied to any shadow cluster $S_{m, m_{1}, \ldots, m_{q}}$ as $m n+m_{1} n_{1}+\cdots+m_{q} n_{q}$, with $E(C)=\left\{n, n_{1}, \ldots, n_{r}=1, \ldots\right\}$ being the multiplicity sequence.

The shadow cluster $S_{m, m_{1}, \ldots, m_{u}}$ associated to the ideal $I_{m, m_{1}, \ldots, m_{j}}$ does not in general coincide with the cluster given by $P, P_{1}, \ldots, P_{j}$ with orders $m, m_{1}, \ldots, m_{j}$.

Example 4.8. Consider the curve $C_{1} \equiv\left\{x_{1}=t^{10}, x_{2}=t^{11}, x_{3}=t^{12}+t^{14}+t^{16}\right\}$ from Example 3.8, with H-N matrix

$$
H=\left(\begin{array}{l|llllllllll}
\mathbf{1} & 0 & 0 & 0 & 0 & 0 & 0 & 0 & 0 & 0 & 1 \\
0 & \mathbf{1} & \mathbf{0} & \mathbf{0} & \mathbf{0} & \mathbf{0} & \mathbf{0} & \mathbf{0} & \mathbf{0} & \mathbf{0} & \mathbf{0} \\
0 & 0 & 1 & 0 & 1 & 0 & 1 & 0 & 0 & 0 & 0
\end{array}\right)
$$

with $\Gamma_{1}=\langle 10,11,12,26\rangle$ and $c_{1}=30$. The shadow cluster of the ideal $I_{2,1,1,1}$ is $S_{2,1,1,1,1,1,1}$, since the element 23 of the semigroup is obtained from $I_{2,2,1}$, the element 24 from $I_{2,2,2}$, and $25 \notin \Gamma_{1}$ since, as we have seen above, there is no conic passing exactly through $\left\{P, P_{1}, \ldots, P_{5}\right\}$, and 26 is obtained from a conic (see Example 3.8) passing through $S_{2,1,1,1,1,1,1}$.

Example 4.9. Consider the curve

$$
C_{2} \equiv\left\{x_{1}=t^{20}, x_{2}=t^{21}, x_{3}=t^{24}+2 t^{27}+t^{29}, x_{4}=t^{26}+3 t^{29}\right\}
$$


from Examples 2.8 and 3.9, which has a 21-column H-N matrix

$$
H=\left(\begin{array}{l|llllllllllll}
\mathbf{1} & 0 & 0 & 0 & 0 & 0 & 0 & 0 & 0 & 0 & 0 & \ldots & 1 \\
0 & \mathbf{1} & \mathbf{0} & \mathbf{0} & \mathbf{0} & \mathbf{0} & \mathbf{0} & \mathbf{0} & \mathbf{0} & \mathbf{0} & \mathbf{0} & \ldots & \mathbf{0} \\
0 & 0 & 0 & 0 & 1 & 0 & 0 & 2 & 0 & 1 & 0 & \ldots & 0 \\
0 & 0 & 0 & 0 & 0 & 0 & 1 & 0 & 0 & 3 & 0 & \ldots & 0
\end{array}\right)
$$

with $\Gamma=\langle 20,21,24,26,77\rangle$ and $E(C)=\{20,1, \ldots, 1\}$. The shadow cluster of the ideal $I_{3,2,2,2,2,2,2,2}$ is $S_{3,2,2,2,2,2,2,2,1,1,1}$ since the elements of the semigroup are greater than $3 \times 20+2 \times 7$, since $75 \notin \Gamma_{2}$, and since 76 is obtained with $x_{3} x_{4}^{2}$. The hypersurface $X_{3} X_{4}^{2}$ passes only through $P_{1}, P_{2}, \ldots, P_{6}$, the element 77 is given by $x_{3}^{3}-x_{1} x_{4}^{2}$, and the cubic $X_{3}^{3}-X_{1} X_{4}^{2}$ passes exactly through $S_{3,2,2,2,2,2,2,2,1,1,1}$.

Proposition 4.10. For any subsequence $\left\{P, P_{1}, \ldots, P_{j}\right\}$ of INPs of a curve $C$, the orders $m, m_{1}, \ldots, m_{j}$ of the ideals $I_{m, m_{1}, \ldots, m_{j}}$ necessary to compute its shadow clusters are bounded by means of the conditions given by the proximity relations, that is, by the multiplicity sequence.

Proof. It is sufficient to consider the bound

$$
c^{*}=n(n-1)+n_{1}\left(n_{1}-1\right)+\cdots+n_{r-1}\left(n_{r-1}-1\right)
$$

for the conductor $c$, obtained in (2-1) from the multiplicity sequence. Since, by (2-2), all the generators of the semigroup $\Gamma(C)$ are at most $c+n-1$, the $m_{i}$ are bounded by $c^{*}$ and the $n_{i}$.

Remark 4.11. For space curves, the approximate roots $F_{i}$ considered in Remark 4.3 have to be chosen among the hypersurfaces corresponding to sequences $\left\{m, m_{1}, \ldots, m_{j}\right\}$ of shadow clusters of the INPs.

Lastly, we consider a subsemigroup of the semigroup of values determined by the special $*$-simple complete ideals of the sequence of INPs associated to the curve.

Lipman $[1988,2.1]$ associates to any sequence $\left\{P, P_{1}, \ldots, P_{j}\right\}$ of INPs a unique special $*$-simple complete ideal $\mathfrak{p}_{j}$, simple for the complete product of ideals $I * J=\overline{I \cdot J}$. This $\mathfrak{m}$-primary ideal is the ideal of all the hypersurfaces which pass exactly through the sequence of INPs and which have orders greater than $B\left(\mathfrak{p}_{j}\right)=\left\{m_{0}^{*}=m^{*}, m_{1}^{*}, \ldots, m_{j}^{*}=1\right\}$. These numbers depend only on the points, and we call them the Lipman multiplicities of the sequence. In [Castellanos 2002], the Lipman multiplicities of some examples are computed.

Given a sequence $\left\{P, P_{1}, \ldots, P_{j}\right\}$ of INPs, there are many sequences of positive integers $\left\{q, q_{1}, \ldots, q_{j}\right\}$ upon which an ideal $I$ is exactly supported, and having those integers as $B(I)$. The Lipman multiplicities of the special $*$-simple complete ideal $\mathfrak{p}_{j}$ form the minimum such sequence for the inverse lexicographic order. 
Consider the sequence $\left\{P, P_{1}, \ldots, P_{s}\right\}$ which determines the curve $C$ and the *-simple complete ideals $\mathfrak{p}_{i}$, for $i \leq j$. Associated to the Lipman multiplicities of $\mathfrak{p}_{i}$, we consider the semigroup of values formed by means of Noether's formula as follows. Consider, for any sequence $\left\{P, P_{1}, \ldots, P_{j}\right\}$, the values

$$
\eta_{j}=m^{(j)} n+m_{1}^{(j)} n_{1}+\cdots+m_{j}^{(j)} n_{j}
$$

where $\left\{n, n_{1}, \ldots, n_{r}=1, \ldots\right\}$ is the multiplicity sequence of $C$ and the orders $\left\{m^{(j)}, m_{1}^{(j)}, \ldots, m_{j}^{(j)}\right\}$ are the Lipman multiplicities for $\mathfrak{p}_{i}$ associated to the points $\left\{P, P_{1}, \ldots, P_{j}\right\}$.

Definition 4.12. The idealistic semigroup for the curve $C$ is the semigroup $\Gamma^{*}(C)=$ $\left\langle\eta_{0}=n, \eta_{1}, \ldots, \eta_{s}\right\rangle$.

For a plane curve singularity $C$, the idealistic semigroup $\Gamma^{*}(C)$ coincides with the semigroup of values of $C$. This follows from the fact that, for any plane curve singularity, the Lipman multiplicities for the ideal $\mathfrak{p}_{m}$, coincide with the multiplicity sequence of the curve; see [Hoskin 1956]. So $\Gamma^{*}(C)$ is generated by

$$
\eta_{j}=n^{(j)} n+n_{1}^{(j)} n_{1}+\cdots+n_{j}^{(j)} n_{j}
$$

where $0 \leq j \leq s$ and $\left\{n^{(j)}, n_{1}^{(j)}, \ldots, n_{j}^{(j)}=1\right\}$ is the multiplicity sequence of the curve associated to the points $\left\{P, P_{1}, \ldots, P_{j}\right\}$, that is, a curve having $\eta_{j}$ as intersection multiplicity with $C$ at $P$. Then the generators of the semigroup of the curve $\left\{\bar{\beta}_{0}=n, \bar{\beta}_{1}, \ldots, \bar{\beta}_{g}\right\}$ correspond to the intersection multiplicity of the maximal contact curves $\Phi_{i}$ of genus $i$, that is,

$$
\bar{\beta}_{i}=n^{\left(s_{i}\right)} n+n_{1}^{\left(s_{i}\right)} n_{1}+\cdots+n_{s_{i}}^{\left(s_{i}\right)} n_{s_{i}}
$$

where $\left\{n^{\left(s_{i}\right)}, n_{1}^{\left(s_{i}\right)}, \ldots, n_{s_{i}}^{\left(s_{i}\right)}=1\right\}$ is the multiplicity sequence of $\Phi_{i}$ [Campillo 1980, Proposition 4.3.4], and so $\bar{\beta}_{i} \in \Gamma^{*}(C)$.

Corollary 4.13. The idealistic semigroup of a curve $C$ is $\Gamma^{*}(C) \subset \Gamma(C)$.

Observe that the two semigroups are different in general.

Example 4.14. Consider the curve $C \equiv\left\{x_{1}=t^{6}, x_{2}=t^{7}, x_{3}=t^{8}+t^{11}\right\}$, with H-N matrix

$$
H=\left(\begin{array}{l|llllll}
\mathbf{1} & 0 & 0 & 0 & 0 & 0 & 1 \\
0 & \mathbf{1} & \mathbf{0} & \mathbf{0} & \mathbf{0} & \mathbf{0} & \mathbf{0} \\
0 & 0 & 1 & 0 & 0 & 1 & 0
\end{array}\right)
$$


semigroup $\Gamma(C)=\langle 6,7,8,17\rangle$, and $E(C)=\{6,1\}$. The Lipman $*$-special ideals $\mathfrak{p}_{i}$ associated to $\left\{P, P_{1}, \ldots, P_{i}\right\}$ are:

$\begin{array}{llr}\text { Ideals } & \text { Orders } & \text { Values } \\ \mathfrak{p}_{0}=\overline{(X, Y, Z)} & B\left(\mathfrak{p}_{0}\right)=\{1\} & 6 \\ \mathfrak{p}_{1}=\overline{\left(X^{2}, Y, Z\right)} & B\left(\mathfrak{p}_{1}\right)=\{1,1\} & 7 \\ \mathfrak{p}_{2}=\overline{\left(X^{2}, Y^{2}, Z\right)} & B\left(\mathfrak{p}_{2}\right)=\{1,1,1\} & 8 \\ \mathfrak{p}_{3}=\overline{\left(X^{3}, Y^{2}, Z^{2}, X Z\right)} & B\left(\mathfrak{p}_{3}\right)=\{2,1,1\} & 14 \\ \mathfrak{p}_{4}=\overline{\left(X^{3}, Y^{3}, Z^{2}, X Z-Y^{2}, Y Z\right)} & B\left(\mathfrak{p}_{4}\right)=\{2,1,1,1\} & 16 \\ \mathfrak{p}_{5}=\overline{\left(X^{3}, Y^{3}, Z^{2}, X Z-Y^{2}\right)} & B\left(\mathfrak{p}_{5}\right)=\{2,1,1,1,1\} & 23 \\ \mathfrak{p}_{6}=\overline{\left(X^{4}, Y^{4}, Z^{3}, X^{2} Z-X Y^{2}, Y Z^{2}\right)} & B\left(\mathfrak{p}_{6}\right)=\{3,1,1,1,1,1\} & \\ \mathfrak{p}_{7}=\overline{\left(X^{4}, Y^{4}, Z^{3}, X^{2} Z-X Y^{2}-Y Z^{2}\right)} & B\left(\mathfrak{p}_{7}\right)=\{3,1,1,1,1,1,1\} & 24\end{array}$

Then the idealistic semigroup is $\Gamma^{*}(C)=\langle 6,7,8\rangle$.

\section{Acknowledgements}

I thank Eduardo Casas for our profitable conversations on the subject of this article.

\section{References}

[Abhyankar and Moh 1973a] S. S. Abhyankar and T. T. Moh, "Newton-Puiseux expansion and generalized Tschirnhausen transformation, I”, J. Reine Angew. Math. 260 (1973), 47-83. MR 49 \#2724 Zbl 0272.12102

[Abhyankar and Moh 1973b] S. S. Abhyankar and T. T. Moh, "Newton-Puiseux expansion and generalized Tschirnhausen transformation, II”, J. Reine Angew. Math. 261 (1973), 29-54. MR 49 \#2724 Zbl 0272.12102

[Campillo 1980] A. Campillo, Algebroid curves in positive characteristic, Lecture Notes in Mathematics 813, Springer, Berlin, 1980. MR 82h:14001 Zbl 0451.14010

[Campillo et al. 1996] A. Campillo, , G. Gonzalez-Sprinberg, and M. Lejeune-Jalabert, "Clusters of infinitely near points", Math. Ann. 306:1 (1996), 169-194. MR 97k:14004 Zbl 0853.14002

[Casas-Alvero 2000] E. Casas-Alvero, Singularities of plane curves, London Math. Soc. Lecture Note Series 276, Cambridge University Press, Cambridge, 2000. MR 2003b:14035 Zbl 0967.14018

[Castellanos 1986] J. Castellanos, "A relation between the sequence of multiplicities and the semigroup of values of an algebroid curve”, J. Pure Appl. Algebra 43:2 (1986), 119-127. MR 87m:14005 Zbl 0612.14008

[Castellanos 1995] J. Castellanos, "On the singularities of space curves", pp. 173-191 in Singularity theory (Trieste, 1991), edited by D. T. Lê, World Scientific, River Edge, NJ, 1995. MR 97b:14030 Zbl 0943.14012

[Castellanos 2002] J. Castellanos, "Complete ideals and singularities of space curves", Math. Z. 239:4 (2002), 777-802. MR 2004a:13015 Zbl 1022.13001

[Castellanos and Castellanos 2005] A. Castellanos and J. Castellanos, "Algorithm for the semigroup of a space curve singularity”, Semigroup Forum 70:1 (2005), 44-60. MR 2005i:14029

[Castellanos and Nuñez 1991] J. Castellanos and A. Nuñez, "On the geometry of the sequence of infinitely near points”, Manuscripta Math. 72:1 (1991), 49-66. MR 92g:14004 Zbl 0741.14005 
[Elías 1990] J. Elías, "Characterization of the Hilbert-Samuel polynomials of curve singularities", Compositio Math. 74:2 (1990), 135-155. MR 91h:13016 Zbl 0724.13021

[Goldin and Teissier 2000] R. Goldin and B. Teissier, "Resolving singularities of plane analytic branches with one toric morphism", pp. 315-340 in Resolution of singularities (Obergurgl, 1997), edited by H. Hauser et al., Progress in Math. 181, Birkhäuser, Basel, 2000. MR 2001i:32042 Zbl 0995.14002

[Hoskin 1956] M. A. Hoskin, "Zero-dimensional valuation ideals associated with plane curve branches”, Proc. London Math. Soc. (3) 6 (1956), 70-99. MR 17,665b Zbl 0070.16403

[Lejeune-Jalabert 1973] M. Lejeune-Jalabert, Sur l'équivalence des courbes algébrö̈des planes: Coefficients de Newton - Contribution à l'étude des singularités du point de vue du polygone de Newton, These d'État, Université de Paris VII, Paris, 1973. MR 52 \#800a Zbl 0699.14036

[Lejeune-Jalabert and Teissier 1975] M. Lejeune-Jalabert and B. Teissier, "Clôture integrale des idéaux et équisingularité", in Séminaire sur les singularités (École Polytechnique, Paris, 19741975), Institut Fourier, Grenoble, 1975.

[Lipman 1971] J. Lipman, "Stable ideals and Arf rings", Amer. J. Math. 93 (1971), 649-685. MR 44 \#203 Zbl 0228.13008

[Lipman 1988] J. Lipman, "On complete ideals in regular local rings", pp. 203-231 in Algebraic geometry and commutative algebra: in honor of M. Nagata, vol. I, edited by H. Hijikata et al., Kinokuniya, Tokyo, 1988. MR 90g:14003 Zbl 0693.13011

[Piltant 1997] O. Piltant, "Chains of points infinitely near a regular point on an algebraic variety", Preprint 97-17, École Polytechnique, Paris, 1997.

[Popescu-Pampu 2003] P. Popescu-Pampu, “Approximate roots”, pp. 285-321 in Valuation theory and its applications (Saskatoon, SK, 1999), vol. II, edited by F.-V. Kuhlmann et al., Fields Inst. Commun. 33, Amer. Math. Soc., Providence, RI, 2003. MR 2004k:14006 Zbl 1036.13017

[Teissier 1973] B. Teissier, "Appendix", in Le problème des modules pour les branches planes, by O. Zariski, École Polytechnique, Paris, 1973. Reprinted by Hermann, Paris, 1986. MR 54 \#2662 Zbl 0592.14010

[Zariski 1965] O. Zariski, "Studies in equisingularity, I: Equivalent singularities of plane algebroid curves”, Amer. J. Math. 87 (1965), 507-536. MR 31 \#2243 Zbl 0132.41601

Received November 21, 2003. Revised October 5, 2004.

\section{Julio Castellanos}

Departamento de Algebra

FACUlTad de Matemáticas

UNIVERSIDAD COMPLUTENSE

MADRID 28040

SPAIN

jcaste@mat.ucm.es 